\title{
A new class of analytic functions defined by means of a generalization of the Srivastava-Attiya operator
}

Hari M Srivastava ${ }^{1}$ and Sebastien Gaboury ${ }^{2 *}$

${ }^{*}$ Correspondence:

s1gabour@uqac.ca

${ }^{2}$ Department of Mathematics and

Computer Science, University of

Québec at Chicoutimi, Chicoutimi,

Québec G7H 2B1, Canada

Full list of author information is

available at the end of the article

\begin{abstract}
In this paper, we introduce a new class of analytic functions defined by a new convolution operator $\int_{\left(\lambda_{p}\right),\left(\mu_{q}\right), b}, \lambda$ which generalizes the well-known Srivastava-Attiya operator investigated by Srivastava and Attiya (Integral Transforms Spec. Funct. 18:207-216, 2007). We derive coefficient inequalities, distortion theorems, extreme points and the Fekete-Szegö problem for this new function class.

MSC: Primary 30C45; $11 \mathrm{M} 35$; secondary 30C 10

Keywords: analytic functions; starlike functions; generalized Hurwitz-Lerch zeta function; Srivastava-Attiya operator; Hadamard product
\end{abstract}

\section{Introduction}

Let $\mathcal{A}$ denote the class of functions $f(z)$ normalized by

$$
f(z)=z+\sum_{k=2}^{\infty} a_{k} z^{k}
$$

which are analytic in the open unit disk

$$
\mathbb{U}=\{z: z \in \mathbb{C} \text { and }|z|<1\} .
$$

A function $f(z)$ in the class $\mathcal{A}$ is said to be in the class $\mathcal{S}^{*}(\alpha)$ of starlike functions of order $\alpha$ in $\mathbb{U}$ if it satisfies the following inequality:

$$
\Re\left(\frac{z f^{\prime}(z)}{f(z)}\right)>\alpha \quad(z \in \mathbb{U} ; 0 \leqq \alpha<1)
$$

The largely investigated Srivastava-Attiya operator is defined as [1] (see also [2-4]):

$$
J_{s, a}(f)(z)=z+\sum_{k=2}^{\infty}\left(\frac{1+a}{k+a}\right)^{s} a_{k} z^{k}
$$

where $z \in \mathbb{U}, a \in \mathbb{C} \backslash \mathbb{Z}_{0}^{-}, s \in \mathbb{C}$ and $f \in \mathcal{A}$.

\section{Springer}

C 2015 Srivastava and Gaboury; licensee Springer. This is an Open Access article distributed under the terms of the Creative Commons Attribution License (http://creativecommons.org/licenses/by/4.0), which permits unrestricted use, distribution, and reproduction in any medium, provided the original work is properly credited. 
In fact, the linear operator $J_{s, a}(f)$ can be written as

$$
J_{s, a}(f)(z):=G_{s, a}(z) * f(z)
$$

in terms of the Hadamard product (or convolution), where $G_{s, a}(z)$ is given by

$$
G_{s, a}(z):=(1+a)^{s}\left[\Phi(z, s, a)-a^{-s}\right] \quad(z \in \mathbb{U})
$$

The function $\Phi(z, s, a)$ involved in the right-hand side of (1.5) is the well-known HurwitzLerch zeta function defined by (see, for example, [5, p.121 et seq.]; see also [6] and [7, p.194 et seq.])

$$
\begin{aligned}
\Phi(z, s, a) & :=\sum_{n=0}^{\infty} \frac{z^{n}}{(n+a)^{s}} \\
(a & \left.\in \mathbb{C} \backslash \mathbb{Z}_{0}^{-} ; s \in \mathbb{C} \text { when }|z|<1 ; \Re(s)>1 \text { when }|z|=1\right) .
\end{aligned}
$$

Recently, a new family of $\lambda$-generalized Hurwitz-Lerch zeta functions was investigated by Srivastava [8] (see also [9-13]). Srivastava considered the following function:

$$
\begin{aligned}
\Phi_{\lambda_{1}, \ldots, \lambda_{p} ; \mu_{1}, \ldots, \mu_{q}}^{\left(\rho_{1}, \ldots, \rho_{p}, \sigma_{1}\right)}(z, s, a ; b, \lambda) & \\
= & \frac{1}{\lambda \Gamma(s)} \cdot \sum_{n=0}^{\infty} \frac{\prod_{j=1}^{p}\left(\lambda_{j}\right)_{n \rho_{j}}}{(a+n)^{s} \cdot \prod_{j=1}^{q}\left(\mu_{j}\right)_{n \sigma_{j}}} H_{0,2}^{2,0}\left[(a+n) b^{\frac{1}{\lambda}} \mid \overline{(s, 1),\left(0, \frac{1}{\lambda}\right)}\right] \frac{z^{n}}{n !} \\
& (\min \{\Re(a), \Re(s)\}>0 ; \Re(b)>0 ; \lambda>0),
\end{aligned}
$$

where

$$
\begin{aligned}
& \left(\lambda_{j} \in \mathbb{C}(j=1, \ldots, p) \text { and } \mu_{j} \in \mathbb{C} \backslash \mathbb{Z}_{0}^{-}(j=1, \ldots, q) ; \rho_{j}>0(j=1, \ldots, p) ;\right. \\
& \left.\sigma_{j}>0(j=1, \ldots, q) ; 1+\sum_{j=1}^{q} \sigma_{j}-\sum_{j=1}^{p} \rho_{j} \geqq 0\right)
\end{aligned}
$$

and the equality in the convergence condition holds true for suitably bounded values of $|z|$ given by

$$
|z|<\nabla:=\left(\prod_{j=1}^{p} \rho_{j}^{-\rho_{j}}\right) \cdot\left(\prod_{j=1}^{q} \sigma_{j}^{\sigma_{j}}\right) .
$$

Here, and for the remainder of this paper, $(\lambda)_{\kappa}$ denotes the Pochhammer symbol defined, in terms of the gamma function, by

$$
(\lambda)_{\kappa}:=\frac{\Gamma(\lambda+\kappa)}{\Gamma(\lambda)}= \begin{cases}\lambda(\lambda+1) \cdots(\lambda+n-1) & (\kappa=n \in \mathbb{N} ; \lambda \in \mathbb{C}), \\ 1 & (\kappa=0 ; \lambda \in \mathbb{C} \backslash\{0\}),\end{cases}
$$


it being understood conventionally that $(0)_{0}:=1$ and assumed tacitly that the $\Gamma$-quotient exists (see, for details, [14, p.21 et seq.]).

Definition 1 The $H$-function involved in the right-hand side of (1.7) is the well-known Fox's $H$-function [15, Definition 1.1] (see also [14, 16]) defined by

$$
\begin{aligned}
H_{\mathfrak{p}, \mathfrak{q}}^{m, n}(z) & =H_{\mathfrak{p}, \mathfrak{q}}^{m, n}\left[z \mid \begin{array}{c}
\left(a_{1}, A_{1}\right), \ldots,\left(a_{\mathfrak{p}}, A_{\mathfrak{p}}\right) \\
\left(b_{1}, B_{1}\right), \ldots,\left(b_{\mathfrak{q}}, B_{\mathfrak{q}}\right)
\end{array}\right] \\
& =\frac{1}{2 \pi \mathrm{i}} \int_{\mathcal{L}} \Xi(s) z^{-s} \mathrm{~d} s \quad(z \in \mathbb{C} \backslash\{0\} ;|\arg (z)|<\pi),
\end{aligned}
$$

where

$$
\Xi(s)=\frac{\prod_{j=1}^{m} \Gamma\left(b_{j}+B_{j} s\right) \cdot \prod_{j=1}^{n} \Gamma\left(1-a_{j}-A_{j} s\right)}{\prod_{j=n+1}^{\mathfrak{p}} \Gamma\left(a_{j}+A_{j} s\right) \cdot \prod_{j=m+1}^{\mathfrak{q}} \Gamma\left(1-b_{j}-B_{j} s\right)},
$$

an empty product is interpreted as $1, m, n, \mathfrak{p}$ and $\mathfrak{q}$ are integers such that $1 \leqq m \leqq \mathfrak{q}, 0 \leqq$ $n \leqq \mathfrak{p}, A_{j}>0(j=1, \ldots, \mathfrak{p}), B_{j}>0(j=1, \ldots, \mathfrak{q}), a_{j} \in \mathbb{C}(j=1, \ldots, \mathfrak{p}), b_{j} \in \mathbb{C}(j=1, \ldots, \mathfrak{q})$ and $\mathcal{L}$ is a suitable Mellin-Barnes type contour separating the poles of the gamma functions

$$
\left\{\Gamma\left(b_{j}+B_{j} s\right)\right\}_{j=1}^{m}
$$

from the poles of the gamma functions

$$
\left\{\Gamma\left(1-a_{j}+A_{j} s\right)\right\}_{j=1}^{n}
$$

It is worthy to mention that using the fact that [8, p.1496, Remark 7]

$$
\lim _{b \rightarrow 0}\left\{H_{0,2}^{2,0}\left[(a+n) b^{\frac{1}{\lambda}} \overline{(s, 1),\left(0, \frac{1}{\lambda}\right)}\right]\right\}=\lambda \Gamma(s) \quad(\lambda>0),
$$

equation (1.7) reduces to

$$
\begin{aligned}
\Phi_{\lambda_{1}, \ldots, \lambda_{p} ; \mu_{1}, \ldots, \mu_{q}}^{\left(\rho_{1}, \ldots, \rho_{p}, \sigma_{1}, \ldots, \sigma_{q}\right)}(z, s, a ; 0, \lambda) & :=\Phi_{\lambda_{1}, \ldots, \lambda_{p} ; \mu_{1}, \ldots, \mu_{q}}^{\left(\rho_{1}, \ldots, \rho_{p}, \sigma_{1}, \ldots, \sigma_{q}\right)}(z, s, a) \\
& =\sum_{n=0}^{\infty} \frac{\prod_{j=1}^{p}\left(\lambda_{j}\right)_{n \rho_{j}}}{(a+n)^{s} \cdot \prod_{j=1}^{q}\left(\mu_{j}\right)_{n \sigma_{j}}} \frac{z^{n}}{n !} .
\end{aligned}
$$

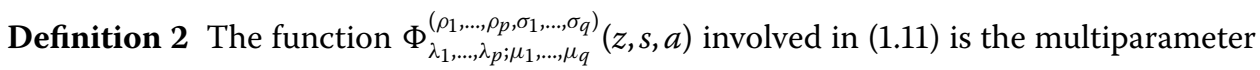
extension and generalization of the Hurwitz-Lerch zeta function $\Phi(z, s, a)$ introduced by Srivastava et al. [13, p.503, Eq. (6.2)] defined by

$$
\begin{aligned}
& \Phi_{\lambda_{1}, \ldots, \lambda_{p} ; \mu_{1}, \ldots, \mu_{q}}^{\left(\rho_{1}, \ldots, \rho_{p}, \sigma_{1}, \ldots, \sigma_{q}\right)}(z, s, a):=\sum_{n=0}^{\infty} \frac{\prod_{j=1}^{p}\left(\lambda_{j}\right)_{n \rho_{j}}}{(a+n)^{s} \cdot \prod_{j=1}^{q}\left(\mu_{j}\right)_{n \sigma_{j}}} \frac{z^{n}}{n !} \\
& \left(p, q \in \mathbb{N}_{0} ; \lambda_{j} \in \mathbb{C}(j=1, \ldots, p) ; a, \mu_{j} \in \mathbb{C} \backslash \mathbb{Z}_{0}^{-}(j=1, \ldots, q) ;\right. \\
& \rho_{j}, \sigma_{k} \in \mathbb{R}^{+}(j=1, \ldots, p ; k=1, \ldots, q) ;
\end{aligned}
$$




$$
\begin{aligned}
& \Delta>-1 \text { when } s, z \in \mathbb{C} ; \\
& \Delta=-1 \text { and } s \in \mathbb{C} \text { when }|z|<\nabla^{*} ; \\
& \left.\Delta=-1 \text { and } \Re(\Xi)>\frac{1}{2} \text { when }|z|=\nabla^{*}\right)
\end{aligned}
$$

with

$$
\begin{aligned}
& \nabla^{*}:=\left(\prod_{j=1}^{p} \rho_{j}^{-\rho_{j}}\right) \cdot\left(\prod_{j=1}^{q} \sigma_{j}^{\sigma_{j}}\right), \\
& \Delta:=\sum_{j=1}^{q} \sigma_{j}-\sum_{j=1}^{p} \rho_{j} \text { and } \Xi:=s+\sum_{j=1}^{q} \mu_{j}-\sum_{j=1}^{p} \lambda_{j}+\frac{p-q}{2} .
\end{aligned}
$$

We propose to consider the following linear operator

$$
J_{\left(\lambda_{p}\right),\left(\mu_{q}\right), b}^{s, a, \lambda}(f): \mathcal{A} \rightarrow \mathcal{A}
$$

defined by

$$
J_{\left(\lambda_{p}\right),\left(\mu_{q}\right), b}^{s, a, \lambda}(f)(z)=G_{\left(\lambda_{p}\right),\left(\mu_{q}\right), b}^{s, a, \lambda}(z) * f(z),
$$

where $*$ denotes the Hadamard product (or convolution) of analytic functions, and the function $G_{\left(\lambda_{p}\right),\left(\mu_{q}\right), b}^{s, a)}(z)$ is given by

$$
\begin{aligned}
G_{\left(\lambda_{p}\right),\left(\mu_{q}\right), b}^{s, a}(z) \\
:=\frac{\lambda \prod_{j=1}^{q}\left(\mu_{j}\right) \Gamma(s)(a+1)^{s}}{\prod_{j=1}^{p}\left(\lambda_{j}\right)} \cdot \Lambda(a+1, b, s, \lambda)^{-1} \\
\\
\cdot\left[\Phi_{\lambda_{1}, \ldots, \lambda_{p} ; \mu_{1}, \ldots, \mu_{q}}^{(1, \ldots, 1,1, \ldots, 1)}(z, s, a ; b, \lambda)-\frac{a^{-s}}{\lambda \Gamma(s)} \Lambda(a, b, s, \lambda)\right] \\
=z+\sum_{k=2}^{\infty} \frac{\prod_{j=1}^{p}\left(\lambda_{j}+1\right)_{k-1}}{\prod_{j=1}^{q}\left(\mu_{j}+1\right)_{k-1}}\left(\frac{a+1}{a+k}\right)^{s}\left(\frac{\Lambda(a+k, b, s, \lambda)}{\Lambda(a+1, b, s, \lambda)}\right) \frac{z^{k}}{k !}
\end{aligned}
$$

with

$$
\Lambda(a, b, s, \lambda):=H_{0,2}^{2,0}\left[a b^{\frac{1}{\lambda}} \overline{(s, 1),\left(0, \frac{1}{\lambda}\right)}\right] .
$$

Combining (1.15) and (1.16), we obtain

$$
\begin{aligned}
& J_{\left(\lambda_{p}\right),\left(\mu_{q}\right), b}^{s, a}(f)(z) \\
& =z+\sum_{k=2}^{\infty} \frac{\prod_{j=1}^{p}\left(\lambda_{j}+1\right)_{k-1}}{\prod_{j=1}^{q}\left(\mu_{j}+1\right)_{k-1}}\left(\frac{a+1}{a+k}\right)^{s}\left(\frac{\Lambda(a+k, b, s, \lambda)}{\Lambda(a+1, b, s, \lambda)}\right) a_{k} \frac{z^{k}}{k !} \\
& \quad\left(\lambda_{j} \in \mathbb{C}(j=1, \ldots, p) \text { and } \mu_{j} \in \mathbb{C} \backslash \mathbb{Z}_{0}^{-}(j=1, \ldots, q) ; p \leqq q+1 ; z \in \mathbb{U}\right),
\end{aligned}
$$


with

$$
\min \{\Re(a), \Re(s)\}>0 ; \quad \lambda>0 \quad \text { if } \Re(b)>0
$$

and

$$
s \in \mathbb{C} ; \quad a \in \mathbb{C} \backslash \mathbb{Z}_{0}^{-} \quad \text { if } b=0 .
$$

Remark 1 It follows from (1.15) and (1.17) that the operator $J_{\left(\lambda_{p}\right),\left(\mu_{q}\right), 0}^{s, a, \lambda}(f)$ (special case of (1.17) when $b=0$ ) can be defined for $a \in \mathbb{C} \backslash \mathbb{Z}^{-}$by the following limit relationship:

$$
J_{\left(\lambda_{p}\right),\left(\mu_{q}\right), 0}^{s, 0, \lambda}(f)(z):=\lim _{a \rightarrow 0}\left\{J_{\left(\lambda_{p}\right),\left(\mu_{q}\right), 0}^{s, a, \lambda}(f)(z)\right\} .
$$

We can see that the operator $J_{\left(\lambda_{p}\right),\left(\mu_{q}\right), b}^{s, a, \lambda}$ generalizes several recently investigated operators such as:

(i) If $p=2, q=1$ and $b=0$, then $J_{\left(\lambda_{1}, \lambda_{2}\right),\left(\mu_{1}\right), 0}^{s, a}=J_{\lambda_{1}, \lambda_{2} ; \mu_{1}}^{s, a}$, where $J_{\lambda_{1}, \lambda_{2} ; \mu_{1}}^{s, a}$ is the linear operator introduced by Prajapat and Bulboacă [17, p.571, Eq. (1.8)].

(ii) $J_{(\gamma-1,1),(v), 0}^{s, a, \lambda}=I_{a, v, \gamma}^{s}$, where $I_{a, v, \gamma}^{s}$ is the generalized operator recently studied by Noor and Bukhari [18, p.2, Eq. (1.3)].

(iii) $J_{(\gamma-1,1),(v), 0}^{0,0, \lambda}=I_{v, \gamma}^{s}$, where $I_{v, \gamma}^{s}$ is the Choi-Saigo-Srivastava operator [19].

(iv) $J_{(\gamma, 1),(\gamma), 0}^{s, a, \lambda}=J_{s, a}$, where $J_{s, a}$ is the Srivastava-Attiya operator [1].

(v) $J_{(\gamma, 1),(\gamma), 0}^{-r, a, \lambda}=I(r, a)(a \geqq 0, r \in \mathbb{Z})$, where the operator $I(r, a)$ is the one introduced by Cho and Srivastava [20].

(vi) $J_{(\beta, 1),(\alpha+\beta), 0}^{0, a, \lambda}=\mathcal{Q}_{\beta}^{\alpha}(\alpha \geqq 0, \beta>-1)$, where the operator $\mathcal{Q}_{\beta}^{\alpha}$ was studied by Jung et al. [21].

(vii) $J_{(\gamma, 1),(\gamma), 0}^{1, a, \lambda}=J_{a}(a \geqq-1)$, where $J_{a}$ denotes the Bernardi operator [22].

(viii) $J_{(\gamma, 1),(v), 0}^{0,0,}=\mathcal{L}(\gamma, v)$, where $\mathcal{L}(\gamma, v)$ is the well-known Carlson-Shaffer operator [23].

(ix) $J_{(2,1),(2-\gamma), 0}^{0,0, \lambda}=\Omega_{z}^{\gamma}(0 \leqq \gamma<1)$, where $\Omega_{z}^{\gamma}$ is the fractional integral operator investigated by Owa and Srivastava [24].

(x) $J_{\left(\lambda_{1}-1, \ldots, \lambda_{p}-1,1\right),\left(\mu_{1}-1, \ldots, \mu_{q}-1,0\right), 0}^{0, \lambda}=H_{1}\left(\lambda_{1}, \ldots, \lambda_{p} ; \mu_{1}, \ldots, \mu_{q}\right)(p \leqq q+1)$, where the operator $H_{1}\left(\lambda_{1}, \ldots, \lambda_{p} ; \mu_{1}, \ldots, \mu_{q}\right)$ is the Dziok-Srivastava operator $[25,26]$ which contains as special cases the Hohlov operator [27] and the Ruscheweyh operator [28].

We say that a function $f \in \mathcal{A}$ is in the class $\mathcal{S}_{\left(\lambda_{p}\right),\left(\mu_{q}\right), b}^{s, a, *)}(\alpha)$ if $J_{\left(\lambda_{p}\right),\left(\mu_{q}\right), b}^{s, a, \lambda}(f)$ is in the class $\mathcal{S}^{*}(\alpha)$, that is, if

$$
\begin{aligned}
& \Re\left(\frac{z\left(J_{\left(\lambda_{p}\right),\left(\mu_{q}\right), b}^{s, a, \lambda}(f)\right)^{\prime}}{J_{\left(\lambda_{p}\right),\left(\mu_{q}\right), b}^{s, a,}(f)}\right)>\alpha \\
& \quad\left(\lambda_{j} \in \mathbb{C}(j=1, \ldots, p) \text { and } \mu_{j} \in \mathbb{C} \backslash \mathbb{Z}_{0}^{-}(j=1, \ldots, q) ;\right. \\
& \quad z \in \mathbb{U} ; 0 \leqq \alpha<1 ; p \leqq q+1),
\end{aligned}
$$

with

$$
\min \{\Re(a), \Re(s)\}>0 ; \quad \lambda>0 \quad \text { if } \Re(b)>0
$$

and

$$
s \in \mathbb{C} ; \quad a \in \mathbb{C} \backslash \mathbb{Z}^{-} \quad \text { if } b=0 .
$$


In this paper, we systematically investigate the class $\mathcal{S}_{\left(\lambda_{p}\right),\left(\mu_{q}\right), b}^{s, a, \lambda^{*}}(\alpha)$ of analytic functions defined above by means of the new generalized Srivastava-Attiya convolution operator $J_{\left(\lambda_{p}\right),\left(\mu_{q}\right), b}^{s, a}$. Especially, we derive coefficient inequalities, distortion theorems, extreme points and the Fekete-Szegö problem for this new function class.

\section{Coefficient inequalities}

Theorem 1 Let $\alpha \in[0,1)$. If $f(z) \in \mathcal{A}$ satisfies the following equality

$$
\sum_{k=2}^{\infty} \frac{(k-\alpha)}{k !}\left|\frac{\prod_{j=1}^{p}\left(\lambda_{j}+1\right)_{k-1}}{\prod_{j=1}^{q}\left(\mu_{j}+1\right)_{k-1}}\right|\left|\left(\frac{a+1}{a+k}\right)^{s} \|\left(\frac{\Lambda(a+k, b, s, \lambda)}{\Lambda(a+1, b, s, \lambda)}\right)\right|\left|a_{k}\right| \leqq 1-\alpha,
$$

then

$$
f \in \mathcal{S}_{\left(\lambda_{p}\right),\left(\mu_{q}\right), b}^{s, a, \lambda, *}(\alpha)
$$

Proof Suppose that inequality (2.1) holds for $\alpha \in[0,1)$. Let us define the function $F(z)$ by

$$
F(z):=\frac{z\left(J_{\left(\lambda_{p}\right),\left(\mu_{q}\right), b}^{s, a, \lambda}\right)^{\prime}(f)(z)}{J_{\left(\lambda_{p}\right),\left(\mu_{q}\right), b}^{s, a}(f)(z)}-\alpha \quad(f(z) \in \mathcal{A})
$$

It is sufficient to prove that

$$
\left|\frac{F(z)-1}{F(z)+1}\right|<1 \quad(z \in \mathbb{U})
$$

to prove that $f(z) \in \mathcal{S}_{(\lambda p),\left(\mu_{q}\right), b}^{s, a), *}(\alpha)$.

In fact, we have that

$$
\begin{aligned}
& |\mathcal{F}(z)|:=\left|\frac{F(z)-1}{F(z)+1}\right|=\left|\frac{\frac{\left.z J_{(\lambda p),\left(\mu_{q}\right), b}^{s, a, \lambda}\right)^{\prime}(f)(z)}{J_{\left(\lambda_{p}\right),\left(\mu_{q}\right), b}^{s, f)(z)}}-\alpha-1}{\frac{\left.z \int_{\left(\lambda_{p}\right),\left(\mu_{q}\right), b}^{s, a,}\right)^{\prime}(f)(z)}{J_{(\lambda,),\left(\mu_{q}\right), b}^{s, a)(z)}}-\alpha+1}\right| \\
& =\left|\frac{\alpha z+\sum_{k=2}^{\infty} \frac{\prod_{j=1}^{p}\left(\lambda_{j}+1\right)_{k-1}}{\prod_{j=1}^{q}\left(\mu_{j}+1\right)_{k-1}}\left(\frac{a+1}{a+k}\right)^{s}\left(\frac{\Lambda(a+k, b, s, \lambda)}{\Lambda(a+1, b, s, \lambda)}\right) \frac{(\alpha+1-k)}{k !} a_{k} z^{k}}{(2-\alpha) z-\sum_{k=2}^{\infty} \frac{\prod_{j=1}^{p}\left(\lambda_{j}+1\right)_{k-1}}{\prod_{j=1}^{q}\left(\mu_{j}+1\right)_{k-1}}\left(\frac{a+1}{a+k}\right)^{s}\left(\frac{\Lambda(a+k, b, s, \lambda)}{\Lambda(a+1, b, s, \lambda)}\right) \frac{(\alpha-1-k)}{k !} a_{k} z^{k}}\right|,
\end{aligned}
$$

and thus

$$
\begin{aligned}
&|\mathcal{F}(z)| \leqq \alpha|z|+\sum_{k=2}^{\infty}\left|\frac{\prod_{j=1}^{p}\left(\lambda_{j}+1\right)_{k-1}}{\prod_{j=1}^{q}\left(\mu_{j}+1\right)_{k-1}}\right|\left|\left(\frac{a+1}{a+k}\right)^{s}\right|\left|\left(\frac{\Lambda(a+k, b, s, \lambda)}{\Lambda(a+1, b, s, \lambda)}\right)\right|\left|\frac{(\alpha+1-k)}{k !}\right|\left|a_{k}\right| \cdot|z|^{k} \\
&(2-\alpha)|z|-\sum_{k=2}^{\infty}\left|\frac{\prod_{j=1}^{p}\left(\lambda_{j}+1\right)_{k-1}}{\prod_{j=1}^{q}\left(\mu_{j}+1\right)_{k-1}}\right|\left|\left(\frac{a+1}{a+k}\right)^{s}\right|\left|\left(\frac{\Lambda(a+k, b, s, \lambda)}{\Lambda(a+1, b, s, \lambda)}\right)\right|\left|\frac{(\alpha-1-k)}{k !}\right|\left|a_{k}\right| \cdot|z|^{k} \\
&<\frac{\alpha+\sum_{k=2}^{\infty}\left|\frac{\prod_{j=1}^{p}\left(\lambda_{j}+1\right)_{k-1}}{\prod_{j=1}^{q}\left(\mu_{j}+1\right)_{k-1}}\right|\left|\left(\frac{a+1}{a+k}\right)^{s}\right|\left|\left(\frac{\Lambda(a+k, b, s, \lambda)}{\Lambda(a+1, b, s, \lambda)}\right)\right| \frac{(k-\alpha-1)}{k !}\left|a_{k}\right|}{(2-\alpha)-\sum_{k=2}^{\infty}\left|\frac{\prod_{j=1}^{p}\left(\lambda_{j}+1\right)_{k-1}}{\prod_{j=1}^{q}\left(\mu_{j}+1\right)_{k-1}}\right|\left|\left(\frac{a+1}{a+k}\right)^{s}\right|\left|\left(\frac{\Lambda(a+k, b, s, \lambda)}{\Lambda(a+1, b, s, \lambda)}\right)\right| \frac{(k-\alpha+1)}{k !}\left|a_{k}\right|} \leqq 1,
\end{aligned}
$$

provided that (2.1) is satisfied. 
The next theorem aims to provide coefficient inequalities for functions $f(z)$ belonging to the class $\mathcal{S}_{\left(\lambda_{p}\right),\left(\mu_{q}\right), b}^{s, \lambda, *}(\alpha)$.

Theorem 2 Let $\alpha \in[0,1)$. Iff $(z) \in \mathcal{S}_{\left(\lambda_{p}\right),\left(\mu_{q}\right), b}^{s, a), \text {, then }}$

$$
\begin{aligned}
\left|a_{k}\right| \leqq & k !\left(\frac{2(1-\alpha)}{k-1}\right)\left|\left(\frac{a+k}{a+1}\right)^{s}\right|\left|\left(\frac{\Lambda(a+1, b, s, \lambda)}{\Lambda(a+k, b, s, \lambda)}\right)\right| \\
& \cdot\left|\frac{\prod_{j=1}^{q}\left(\mu_{j}+1\right)_{k-1}}{\prod_{j=1}^{p}\left(\lambda_{j}+1\right)_{k-1}}\right| \prod_{j=2}^{k-1}\left(1+\frac{2(1-\alpha)}{j-1}\right) \quad(k \in \mathbb{N} \backslash\{1\}) .
\end{aligned}
$$

The result is sharp.

Proof Let

$$
p(z):=\frac{\frac{z\left(\lambda_{(\lambda p),\left(\mu_{q}\right), b}^{s, a, \lambda}\right)^{\prime}(f)(z)}{J_{\left(\lambda_{p}\right),\left(\mu_{q}\right), b}^{(s, f)(z)}}-\alpha}{1-\alpha}=1+c_{1} z+c_{2} z^{2}+\cdots .
$$

Then $p(z)$ is analytic and

$$
p(0)=1 \quad \text { and } \quad \Re(p(z))>0 \quad(z \in \mathbb{U}) .
$$

We note easily that

$$
\left.z\left(J_{\left(\lambda_{p}\right),\left(\mu_{q}\right), b}^{s, a, \lambda}\right)^{\prime}(f)(z)=[(1-\alpha) p(z)+\alpha]\right]_{\left(\lambda_{p}\right),\left(\mu_{q}\right), b}^{s, a, \lambda}(f)(z) .
$$

With the help of (1.17), we find

$$
\begin{aligned}
& \frac{(k-1)}{k !} \frac{\prod_{j=1}^{p}\left(\lambda_{j}+1\right)_{k-1}}{\prod_{j=1}^{q}\left(\mu_{j}+1\right)_{k-1}}\left(\frac{a+1}{a+k}\right)^{s}\left(\frac{\Lambda(a+k, b, s, \lambda)}{\Lambda(a+1, b, s, \lambda)}\right) a_{k} \\
& =(1-\alpha) \cdot\left[c_{k-1}+\sum_{m=2}^{k-1} \frac{\prod_{j=1}^{p}\left(\lambda_{j}+1\right)_{m-1}}{\prod_{j=1}^{q}\left(\mu_{j}+1\right)_{m-1}}\left(\frac{a+1}{a+m}\right)^{s}\left(\frac{\Lambda(a+m, b, s, \lambda)}{\Lambda(a+1, b, s, \lambda)}\right) \frac{a_{m} c_{k-m}}{m !}\right]
\end{aligned}
$$

for $k \in \mathbb{N} \backslash\{1\}$.

By making use of the Carathéodory lemma [29, p.41], we have

$$
\begin{aligned}
& \frac{(k-1)}{k !}\left|\frac{\prod_{j=1}^{p}\left(\lambda_{j}+1\right)_{k-1}}{\prod_{j=1}^{q}\left(\mu_{j}+1\right)_{k-1}}\right|\left|\left(\frac{a+1}{a+k}\right)^{s}\right|\left|\left(\frac{\Lambda(a+k, b, s, \lambda)}{\Lambda(a+1, b, s, \lambda)}\right)\right| \cdot\left|a_{k}\right| \\
& \leqq 2(1-\alpha) \\
& \quad\left[1+\sum_{m=2}^{k-1}\left|\frac{\prod_{j=1}^{p}\left(\lambda_{j}+1\right)_{m-1}}{\prod_{j=1}^{q}\left(\mu_{j}+1\right)_{m-1}}\right|\left|\left(\frac{a+1}{a+m}\right)^{s}\right|\left|\left(\frac{\Lambda(a+m, b, s, \lambda)}{\Lambda(a+1, b, s, \lambda)}\right)\right| \frac{\left|a_{m}\right|}{m !}\right] .
\end{aligned}
$$

We have to prove that inequality (2.4) holds true for $k \in \mathbb{N} \backslash\{1\}$. We will proceed by the principle of mathematical induction. If $k=2$ in (2.6), we obtain

$$
\left|a_{2}\right| \leqq 4(1-\alpha)\left|\frac{\prod_{j=1}^{q}\left(\mu_{j}+1\right)}{\prod_{j=1}^{p}\left(\lambda_{j}+1\right)}\right|\left|\left(\frac{a+2}{a+1}\right)^{s}\right|\left|\left(\frac{\Lambda(a+1, b, s, \lambda)}{\Lambda(a+2, b, s, \lambda)}\right)\right| .
$$


Now suppose that (2.4) is satisfied for $k \leqq n$. Then, from (2.4) and (2.6), we have that

$$
\begin{aligned}
& \frac{n}{(n+1) !}\left|\frac{\prod_{j=1}^{p}\left(\lambda_{j}+1\right)_{n}}{\prod_{j=1}^{q}\left(\mu_{j}+1\right)_{n}}\right|\left|\left(\frac{a+1}{a+n+1}\right)^{s}\right|\left|\left(\frac{\Lambda(a+n+1, b, s, \lambda)}{\Lambda(a+1, b, s, \lambda)}\right)\right| \cdot\left|a_{n+1}\right| \\
& \quad \leqq 2(1-\alpha)\left[1+\sum_{m=2}^{n}\left|\frac{\prod_{j=1}^{p}\left(\lambda_{j}+1\right)_{m-1}}{\prod_{j=1}^{q}\left(\mu_{j}+1\right)_{m-1}}\right|\left|\left(\frac{a+1}{a+m}\right)^{s}\right|\left|\left(\frac{\Lambda(a+m, b, s, \lambda)}{\Lambda(a+1, b, s, \lambda)}\right)\right| \frac{\left|a_{m}\right|}{m !}\right] \\
& \quad \leqq 2(1-\alpha)\left[1+\sum_{m=2}^{n} \frac{2(1-\alpha)}{m-1} \prod_{j=2}^{m-1}\left(1+\frac{2(1-\alpha)}{j-1}\right)\right] \\
& \quad \leqq 2(1-\alpha) \prod_{j=2}^{n}\left(1+\frac{2(1-\alpha)}{j-1}\right),
\end{aligned}
$$

whence

$$
\begin{aligned}
\left|a_{k}\right| \leqq & k !\left(\frac{2(1-\alpha)}{k-1}\right)\left|\left(\frac{a+k}{a+1}\right)^{s}\right|\left|\left(\frac{\Lambda(a+1, b, s, \lambda)}{\Lambda(a+k, b, s, \lambda)}\right)\right| \\
\cdot & \left|\frac{\prod_{j=1}^{q}\left(\mu_{j}+1\right)_{k-1}}{\prod_{j=1}^{p}\left(\lambda_{j}+1\right)_{k-1}}\right| \prod_{j=2}^{k-1}\left(1+\frac{2(1-\alpha)}{j-1}\right) \quad(k \in \mathbb{N} \backslash\{1\}) .
\end{aligned}
$$

The result is sharp for the function $f(z)$ given by

$$
\begin{aligned}
f(z)= & z+\frac{2(1-\alpha)}{k-1}\left(\frac{a+k}{a+1}\right)^{s}\left(\frac{\Lambda(a+1, b, s, \lambda)}{\Lambda(a+k, b, s, \lambda)}\right) \\
& \cdot \frac{\prod_{j=1}^{q}\left(\mu_{j}+1\right)_{k-1}}{\prod_{j=1}^{p}\left(\lambda_{j}+1\right)_{k-1}} \prod_{j=2}^{k-1}\left(1+\frac{2(1-\alpha)}{j-1}\right) z^{k} \quad(k \in \mathbb{N} \backslash\{1\}) .
\end{aligned}
$$

3 Distortion inequalities for the function class $\mathcal{S}_{\left(\lambda_{p}\right),\left(\mu_{q}\right), b}^{s, \lambda, *}(\alpha)$

In this section, we establish distortion inequalities for functions belonging to the class $\mathcal{S}_{\left(\lambda_{p}\right),\left(\mu_{q}\right), b}^{s, a, \lambda}(\alpha)$. These inequalities are given in the following theorem.

Theorem 3 Let $f(z) \in \mathcal{S}_{\left(\lambda_{p}\right),\left(\mu_{q}\right), b}^{s, a, \lambda, *}(\alpha)$ and $0 \leqq \alpha<1$. Then

$$
\begin{aligned}
& r-2(1-\alpha) r^{2} \sum_{k=2}^{\infty} \frac{k !}{k-1}\left|\left(\frac{a+k}{a+1}\right)^{s}\right|\left|\left(\frac{\Lambda(a+1, b, s, \lambda)}{\Lambda(a+k, b, s, \lambda)}\right)\right| \\
& \quad \cdot\left|\frac{\prod_{j=1}^{q}\left(\mu_{j}+1\right)_{k-1}}{\prod_{j=1}^{p}\left(\lambda_{j}+1\right)_{k-1}}\right| \prod_{j=2}^{k-1}\left(1+\frac{2(1-\alpha)}{j-1}\right) \\
& \leqq|f(z)| \\
& \leqq+2(1-\alpha) r^{2} \sum_{k=2}^{\infty} \frac{k !}{k-1}\left|\left(\frac{a+k}{a+1}\right)^{s}\right|\left|\left(\frac{\Lambda(a+1, b, s, \lambda)}{\Lambda(a+k, b, s, \lambda)}\right)\right| \\
& \cdot\left|\frac{\prod_{j=1}^{q}\left(\mu_{j}+1\right)_{k-1}}{\prod_{j=1}^{p}\left(\lambda_{j}+1\right)_{k-1}}\right| \prod_{j=2}^{k-1}\left(1+\frac{2(1-\alpha)}{j-1}\right) \quad(|z|=r<1)
\end{aligned}
$$


and

$$
\begin{aligned}
1-2(1-\alpha) r \sum_{k=2}^{\infty} \frac{k \cdot k !}{k-1}\left|\left(\frac{a+k}{a+1}\right)^{s}\right|\left|\left(\frac{\Lambda(a+1, b, s, \lambda)}{\Lambda(a+k, b, s, \lambda)}\right)\right| \\
\cdot\left|\frac{\prod_{j=1}^{q}\left(\mu_{j}+1\right)_{k-1}}{\prod_{j=1}^{p}\left(\lambda_{j}+1\right)_{k-1}}\right| \prod_{j=2}^{k-1}\left(1+\frac{2(1-\alpha)}{j-1}\right) \\
\leqq\left|f^{\prime}(z)\right| \\
\leqq 1+2(1-\alpha) r \sum_{k=2}^{\infty} \frac{k \cdot k !}{k-1}\left|\left(\frac{a+k}{a+1}\right)^{s}\right|\left|\left(\frac{\Lambda(a+1, b, s, \lambda)}{\Lambda(a+k, b, s, \lambda)}\right)\right| \\
\quad \cdot \frac{\prod_{j=1}^{q}\left(\mu_{j}+1\right)_{k-1}}{\prod_{j=1}^{p}\left(\lambda_{j}+1\right)_{k-1}} \mid \prod_{j=2}^{k-1}\left(1+\frac{2(1-\alpha)}{j-1}\right) \quad(|z|=r<1) .
\end{aligned}
$$

Proof Let $f(z) \in \mathcal{A}$ be given by (1.1). Then, making use of Theorem 2, we find

$$
\begin{aligned}
|f(z)| \leqq & |z|+\sum_{k=2}^{\infty}\left|a_{k}\right| \cdot\left|z^{k}\right| \\
\leqq & +2(1-\alpha) r^{2} \sum_{k=2}^{\infty} \frac{k !}{k-1}\left|\left(\frac{a+k}{a+1}\right)^{s}\right|\left|\left(\frac{\Lambda(a+1, b, s, \lambda)}{\Lambda(a+k, b, s, \lambda)}\right)\right| \\
& \cdot\left|\frac{\prod_{j=1}^{q}\left(\mu_{j}+1\right)_{k-1}}{\prod_{j=1}^{p}\left(\lambda_{j}+1\right)_{k-1}}\right| \prod_{j=2}^{k-1}\left(1+\frac{2(1-\alpha)}{j-1}\right) \quad(|z|=r<1)
\end{aligned}
$$

and

$$
\begin{aligned}
|f(z)| \geqq|z|-\sum_{k=2}^{\infty}\left|a_{k}\right| \cdot\left|z^{k}\right| \\
\geqq r-2(1-\alpha) r^{2} \sum_{k=2}^{\infty} \frac{k !}{k-1}\left|\left(\frac{a+k}{a+1}\right)^{s}\right|\left|\left(\frac{\Lambda(a+1, b, s, \lambda)}{\Lambda(a+k, b, s, \lambda)}\right)\right| \\
\cdot\left|\frac{\prod_{j=1}^{q}\left(\mu_{j}+1\right)_{k-1}}{\prod_{j=1}^{p}\left(\lambda_{j}+1\right)_{k-1}}\right| \prod_{j=2}^{k-1}\left(1+\frac{2(1-\alpha)}{j-1}\right) \quad(|z|=r<1) .
\end{aligned}
$$

From (1.1), we also have that

$$
\begin{aligned}
\left|f^{\prime}(z)\right| \leqq & 1+\sum_{k=2}^{\infty} k \cdot\left|a_{k}\right| \cdot\left|z^{k-1}\right| \\
\leqq & 1+2(1-\alpha) r \sum_{k=2}^{\infty} \frac{k \cdot k !}{k-1}\left|\left(\frac{a+k}{a+1}\right)^{s}\right|\left|\left(\frac{\Lambda(a+1, b, s, \lambda)}{\Lambda(a+k, b, s, \lambda)}\right)\right| \\
& \cdot\left|\frac{\prod_{j=1}^{q}\left(\mu_{j}+1\right)_{k-1}}{\prod_{j=1}^{p}\left(\lambda_{j}+1\right)_{k-1}}\right| \prod_{j=2}^{k-1}\left(1+\frac{2(1-\alpha)}{j-1}\right) \quad(|z|=r<1)
\end{aligned}
$$


and

$$
\begin{aligned}
\left|f^{\prime}(z)\right| \geqq & -\sum_{k=2}^{\infty} k \cdot\left|a_{k}\right| \cdot\left|z^{k}\right| \\
\geqq & 1-2(1-\alpha) r \sum_{k=2}^{\infty} \frac{k \cdot k !}{k-1}\left|\left(\frac{a+k}{a+1}\right)^{s}\right|\left|\left(\frac{\Lambda(a+1, b, s, \lambda)}{\Lambda(a+k, b, s, \lambda)}\right)\right| \\
& \cdot\left|\frac{\prod_{j=1}^{q}\left(\mu_{j}+1\right)_{k-1}}{\prod_{j=1}^{p}\left(\lambda_{j}+1\right)_{k-1}}\right| \prod_{j=2}^{k-1}\left(1+\frac{2(1-\alpha)}{j-1}\right) \quad(|z|=r<1) .
\end{aligned}
$$

We thus obtain the results (3.1) and (3.2) asserted by Theorem 3 .

\section{Extreme points}

This section is devoted to presenting the extreme points of the function class $\mathcal{S}_{\left(\lambda_{p}\right),\left(\mu_{q}\right), b}^{s, a, \lambda^{\prime}}(\alpha)$. Let $\widetilde{\mathcal{S}}_{\left(\lambda_{p}\right),\left(\mu_{q}\right), b}^{s, a, \lambda_{0}}$ be the subclass of $\mathcal{S}_{\left(\lambda_{p}\right),\left(\mu_{q}\right), b}^{s, a, \lambda)},(\alpha)$ that consists in all functions $f(z) \in \mathcal{A}$, which satisfy inequality (2.1). Then the extreme points of $\widetilde{\mathcal{S}}_{\left(\lambda_{p}\right),\left(\mu_{q}\right), b}^{s, \lambda)}(\alpha)$ are given by the following theorem.

Theorem 4 Let

$$
f_{1}(z):=z
$$

and

$$
\begin{aligned}
& f_{k}(z):=z+\frac{k !(1-\alpha)}{(k-\alpha)}\left|\frac{\prod_{j=1}^{p}\left(\mu_{j}+1\right)_{k-1}}{\prod_{j=1}^{q}\left(\lambda_{j}+1\right)_{k-1}}\right|\left|\left(\frac{a+k}{a+1}\right)^{s}\right|\left|\left(\frac{\Lambda(a+1, b, s, \lambda)}{\Lambda(a+k, b, s, \lambda)}\right)\right| z^{k} \\
& (k \in \mathbb{N} \backslash\{1\}) .
\end{aligned}
$$

Then

$$
f(z) \in \widetilde{\mathcal{S}}_{\left(\lambda_{p}\right),\left(\mu_{q}\right), b}^{s, \lambda, *}(\alpha) \quad(0 \leqq \alpha<1)
$$

if and only if it can be expressed in the following form:

$$
f(z)=\sum_{k=1}^{\infty} \gamma_{k} f_{k}(z) \quad\left(\gamma_{k}>0 ; \sum_{k=1}^{\infty} \gamma_{k}=1\right) .
$$

Proof Suppose that

$$
\begin{aligned}
f(z)= & \sum_{k=1}^{\infty} \gamma_{k} f_{k}(z) \\
= & z+\sum_{k=2}^{\infty} \gamma_{k} \frac{k !(1-\alpha)}{(k-\alpha)}\left|\frac{\prod_{j=1}^{p}\left(\mu_{j}+1\right)_{k-1}}{\prod_{j=1}^{q}\left(\lambda_{j}+1\right)_{k-1}}\right| \\
& \cdot\left|\left(\frac{a+k}{a+1}\right)^{s}\right|\left|\frac{\Lambda(a+1, b, s, \lambda)}{\Lambda(a+k, b, s, \lambda)}\right| z^{k} .
\end{aligned}
$$


Then

$$
\begin{aligned}
& \sum_{k=2}^{\infty} \frac{(k-\alpha)}{k !}\left|\frac{\prod_{j=1}^{p}\left(\lambda_{j}+1\right)_{k-1}}{\prod_{j=1}^{q}\left(\mu_{j}+1\right)_{k-1}}\right|\left|\left(\frac{a+1}{a+k}\right)^{s}\right|\left|\left(\frac{\Lambda(a+k, b, s, \lambda)}{\Lambda(a+1, b, s, \lambda)}\right)\right| \\
& \cdot \gamma_{k} \frac{k !(1-\alpha)}{(k-\alpha)}\left|\frac{\prod_{j=1}^{p}\left(\mu_{j}+1\right)_{k-1}}{\prod_{j=1}^{q}\left(\lambda_{j}+1\right)_{k-1}}\right|\left|\left(\frac{a+k}{a+1}\right)^{s}\right|\left|\frac{\Lambda(a+1, b, s, \lambda)}{\Lambda(a+k, b, s, \lambda)}\right| \\
& =(1-\alpha) \sum_{k=2}^{\infty} \gamma_{k}=(1-\alpha)\left(1-\gamma_{1}\right) \\
& \leq 1-\alpha .
\end{aligned}
$$

Thus, by the definition of the function class $\widetilde{\mathcal{S}}_{\left(\lambda_{p}\right),\left(\mu_{q}\right), b}^{s, a, \lambda)}$, we have

$$
f \in \widetilde{\mathcal{S}}_{\left(\lambda_{p}\right),\left(\mu_{q}\right), b}^{s, \lambda, *}(\alpha) \quad(0 \leqq \alpha<1)
$$

Conversely, if

$$
f \in \widetilde{\mathcal{S}}_{\left(\lambda_{p}\right),\left(\mu_{q}\right), b}^{s, a, *,}(\alpha) \quad(0 \leqq \alpha<1)
$$

then, by using (2.1), we may set

$$
\begin{aligned}
& \gamma_{k}=\frac{(k-\alpha)}{(1-\alpha) k !}\left|\frac{\prod_{j=1}^{p}\left(\lambda_{j}+1\right)_{k-1}}{\prod_{j=1}^{q}\left(\mu_{j}+1\right)_{k-1}}\right|\left|\left(\frac{a+1}{a+k}\right)^{s}\right|\left|\left(\frac{\Lambda(a+k, b, s, \lambda)}{\Lambda(a+1, b, s, \lambda)}\right)\right|\left|a_{k}\right| \\
& \quad(k \in \mathbb{N} \backslash\{1\}),
\end{aligned}
$$

which implies that

$$
f(z)=\sum_{k=1}^{\infty} \gamma_{k} f_{k} .
$$

The proof of Theorem 4 is thus completed.

\section{The Fekete-Szegö problem}

In this section, we shall obtain the Fekete-Szegö inequality for functions in the class $\mathcal{S}_{\left(\lambda_{p}\right),\left(\mu_{q}\right), b}^{s, a, *}(\alpha)$ when

$$
s>0, \quad a>0 \text { and } 0 \leqq \alpha<1 .
$$

We need to recall an important lemma due to Ma and Minda [30] in order to prove our result involving Fekete-Szegö inequality.

\section{Lemma 1 If}

$$
p(z)=1+c_{1} z+c_{2} z^{2}+\cdots
$$


is an analytic function in $\mathbb{U}$ such that

$$
\Re(p(z))>0 \quad(z \in \mathbb{U}),
$$

then

$$
\left|c_{2}-v c_{1}^{2}\right| \leqq \begin{cases}-4 v+2 & (v \leqq 0) \\ 2 & (0 \leqq v \leqq 1) \\ 4 v-2 & (v \geqq 1)\end{cases}
$$

When $v<0$ or $v>1$, the equality holds true if and only if

$$
p(z)=\frac{1+z}{1-z}
$$

or one of its rotations. If $0<v<1$, then the equality holds true if and only if

$$
p(z)=\frac{1+z^{2}}{1-z^{2}}
$$

or one of its rotations. If $v=0$, then the equality holds true if and only if

$$
p(z)=\left(\frac{1+\omega}{2}\right)\left(\frac{1+z}{1-z}\right)+\left(\frac{1-\omega}{2}\right)\left(\frac{1-z}{1+z}\right) \quad(0 \leqq \omega \leqq 1)
$$

or one of its rotations. If $v=1$, then the equality holds true if and only if $p(z)$ is the reciprocal of one of the functions such that the equality holds true in the case $v=0$.

\section{Theorem 5 Let}

$$
s>0, \quad a>0, \quad 0 \leqq \alpha<1
$$

and

$$
\lambda_{j}>-1 \quad(j=1, \ldots, p), \quad \mu_{j}>-1 \quad(j=1, \ldots, q) .
$$

Iff $\in \mathcal{S}_{\left(\lambda_{p}\right),\left(\mu_{q}\right), b}^{s, a, *}(\alpha)$, then

$$
\left|a_{3}-\tau a_{2}^{2}\right| \leqq\left\{\begin{array}{cc}
3(1-\alpha) \frac{\prod_{j=1}^{q}\left(\mu_{j}+1\right)_{2}}{\prod_{j=1}^{p}\left(\lambda_{j}+1\right)_{2}} & \\
\cdot\left(\frac{a+3}{a+1}\right)^{s}\left(\frac{\Lambda(a+1, b, s, \lambda)}{\Lambda(a+3, b, s, \lambda)}\right)(-4 v+2) & \left(\tau \leqq \sigma_{1}\right), \\
6(1-\alpha) \frac{\prod_{j=1}^{q}\left(\mu_{j}+1\right)_{2}}{\prod_{j=1}^{p}\left(\lambda_{j}+1\right)_{2}} & \left(\sigma_{1} \leqq \tau \leqq \sigma_{2}\right), \\
\cdot\left(\frac{a+3}{a+1}\right)^{s}\left(\frac{\Lambda(a+1, b, s, \lambda)}{\Lambda(a+3, b, s, \lambda)}\right) & \\
3(1-\alpha) \frac{\prod_{j=1}^{q}\left(\mu_{j}+1\right)_{2}}{\prod_{j=1}^{p}\left(\lambda_{j}+1\right)_{2}} & \\
\cdot\left(\frac{a+3}{a+1}\right)^{s}\left(\frac{\Lambda(a+1, b, s, \lambda)}{\Lambda(a+3, b, s, \lambda)}\right)(4 v-2) & \left(\tau \geqq \sigma_{2}\right),
\end{array}\right.
$$


where

$$
\begin{aligned}
v:= & (1-\alpha)\left(\frac{4 \tau}{3} \prod_{j=1}^{p}\left(\frac{\lambda_{j}+2}{\lambda_{j}+1}\right) \prod_{j=1}^{q}\left(\frac{\mu_{j}+1}{\mu_{j}+2}\right)\left(\frac{a+2}{a+3}\right)^{s}\left(\frac{a+2}{a+1}\right)^{s}\right. \\
& \left.\cdot\left(\frac{\Lambda(a+3, b, s, \lambda)}{\Lambda(a+2, b, s, \lambda)}\right)\left(\frac{\Lambda(a+1, b, s, \lambda)}{\Lambda(a+2, b, s, \lambda)}\right)-1\right) \\
\sigma_{1}= & \frac{3}{4} \prod_{j=1}^{p}\left(\frac{\lambda_{j}+1}{\lambda_{j}+2}\right) \prod_{j=1}^{q}\left(\frac{\mu_{j}+2}{\mu_{j}+1}\right)\left(\frac{a+1}{a+2}\right)^{s} \\
& \cdot\left(\frac{\Lambda(a+2, b, s, \lambda)}{\Lambda(a+3, b, s, \lambda)}\right)\left(\frac{\Lambda(a+2, b, s, \lambda)}{\Lambda(a+1, b, s, \lambda)}\right)
\end{aligned}
$$

and

$$
\begin{gathered}
\sigma_{2}=\frac{3(2-\alpha)}{4(1-\alpha)} \prod_{j=1}^{p}\left(\frac{\lambda_{j}+1}{\lambda_{j}+2}\right) \prod_{j=1}^{q}\left(\frac{\mu_{j}+2}{\mu_{j}+1}\right)\left(\frac{a+1}{a+2}\right)^{s} \\
\cdot\left(\frac{\Lambda(a+2, b, s, \lambda)}{\Lambda(a+3, b, s, \lambda)}\right)\left(\frac{\Lambda(a+2, b, s, \lambda)}{\Lambda(a+1, b, s, \lambda)}\right) .
\end{gathered}
$$

The result is sharp.

Proof For $f \in \mathcal{S}_{\left(\lambda_{p}\right),\left(\mu_{q}\right), b}^{s, a), \text { let }}$

$$
p(z)=\frac{\frac{\left.z U_{(\lambda p),(\mu q), b}^{s, a, \lambda}\right)^{\prime}(f)(z)}{\int_{(\lambda p),\left(\mu_{q}\right), b}^{s, a)}(f)(z)}-\alpha}{1-\alpha}=1+c_{1} z+c_{2} z^{2}+\cdots
$$

Then, with the help of (2.5), we have

$$
a_{2}=2(1-\alpha) c_{1} \frac{\prod_{j=1}^{q}\left(\mu_{j}+1\right)}{\prod_{j=1}^{p}\left(\lambda_{j}+1\right)}\left(\frac{a+2}{a+1}\right)^{s}\left(\frac{\Lambda(a+1, b, s, \lambda)}{\Lambda(a+2, b, s, \lambda)}\right)
$$

and

$$
a_{3}=3(1-\alpha) \frac{\prod_{j=1}^{q}\left(\mu_{j}+1\right)_{2}}{\prod_{j=1}^{p}\left(\lambda_{j}+1\right)_{2}}\left(\frac{a+3}{a+1}\right)^{s}\left(\frac{\Lambda(a+1, b, s, \lambda)}{\Lambda(a+3, b, s, \lambda)}\right)\left[c_{2}+(1-\alpha) c_{1}^{2}\right] .
$$

Therefore, we find

$$
\begin{aligned}
a_{3}-\tau a_{2}^{2}= & 3(1-\alpha) \frac{\prod_{j=1}^{q}\left(\mu_{j}+1\right)_{2}}{\prod_{j=1}^{p}\left(\lambda_{j}+1\right)_{2}}\left(\frac{a+3}{a+1}\right)^{s}\left(\frac{\Lambda(a+1, b, s, \lambda)}{\Lambda(a+3, b, s, \lambda)}\right)\left[c_{2}+(1-\alpha) c_{1}^{2}\right] \\
& -4 \tau(1-\alpha)^{2} c_{1}^{2} \frac{\prod_{j=1}^{q}\left(\mu_{j}+1\right)^{2}}{\prod_{j=1}^{p}\left(\lambda_{j}+1\right)^{2}}\left(\frac{a+2}{a+1}\right)^{2 s}\left(\frac{\Lambda(a+1, b, s, \lambda)}{\Lambda(a+2, b, s, \lambda)}\right)^{2} \\
= & 3(1-\alpha) \frac{\prod_{j=1}^{q}\left(\mu_{j}+1\right)_{2}}{\prod_{j=1}^{p}\left(\lambda_{j}+1\right)_{2}}\left(\frac{a+3}{a+1}\right)^{s}\left(\frac{\Lambda(a+1, b, s, \lambda)}{\Lambda(a+3, b, s, \lambda)}\right)\left[c_{2}-c_{1}^{2}(1-\alpha)\right.
\end{aligned}
$$




$$
\begin{aligned}
& \cdot\left(\frac{4 \tau}{3} \prod_{j=1}^{p}\left(\frac{\lambda_{j}+2}{\lambda_{j}+1}\right) \prod_{j=1}^{q}\left(\frac{\mu_{j}+1}{\mu_{j}+2}\right)\left(\frac{a+2}{a+3}\right)^{s}\left(\frac{a+2}{a+1}\right)^{s}\right. \\
& \left.\left.\cdot\left(\frac{\Lambda(a+3, b, s, \lambda)}{\Lambda(a+2, b, s, \lambda)}\right)\left(\frac{\Lambda(a+1, b, s, \lambda)}{\Lambda(a+2, b, s, \lambda)}\right)-1\right)\right] .
\end{aligned}
$$

We thus write

$$
a_{3}-\tau a_{2}^{2}=3(1-\alpha) \frac{\prod_{j=1}^{q}\left(\mu_{j}+1\right)_{2}}{\prod_{j=1}^{p}\left(\lambda_{j}+1\right)_{2}}\left(\frac{a+3}{a+1}\right)^{s}\left(\frac{\Lambda(a+1, b, s, \lambda)}{\Lambda(a+3, b, s, \lambda)}\right)\left(c_{2}-v c_{1}^{2}\right),
$$

where

$$
\begin{gathered}
v:=(1-\alpha)\left(\frac{4 \tau}{3} \prod_{j=1}^{p}\left(\frac{\lambda_{j}+2}{\lambda_{j}+1}\right) \prod_{j=1}^{q}\left(\frac{\mu_{j}+1}{\mu_{j}+2}\right)\left(\frac{a+2}{a+3}\right)^{s}\left(\frac{a+2}{a+1}\right)^{s}\right. \\
\left.\cdot\left(\frac{\Lambda(a+3, b, s, \lambda)}{\Lambda(a+2, b, s, \lambda)}\right)\left(\frac{\Lambda(a+1, b, s, \lambda)}{\Lambda(a+2, b, s, \lambda)}\right)-1\right) .
\end{gathered}
$$

The result asserted by Theorem 5 follows by applying Lemma 1 .

Moreover, if $\tau<\sigma_{1}$ or $\tau>\sigma_{2}$, then the equality holds true if and only if

$$
J_{\left(\lambda_{p}\right),\left(\tau_{q}\right), b}^{s, a, \lambda}(f)(z)=\frac{z}{\left(1-\mathrm{e}^{\mathrm{i} \theta} z\right)^{2(1-\alpha)}} \quad(\theta \in \mathbb{R}) .
$$

For $\sigma_{1}<\tau<\sigma_{2}$, the equality holds true if and only if

$$
J_{\left(\lambda_{p}\right),\left(\tau_{q}\right), b}^{s, a, \lambda}(f)(z)=\frac{z}{\left(1-\mathrm{e}^{\mathrm{i} \theta} z\right)^{1-\alpha}} \quad(\theta \in \mathbb{R}) .
$$

If $\tau=\sigma_{1}$, then the equality holds true if and only if

$$
\begin{aligned}
J_{(\lambda p),\left(\tau_{q}\right), b}^{s, a, \lambda}(f)(z) & =\left(\frac{z}{\left(1-\mathrm{e}^{\mathrm{i} \theta} z\right)^{2(1-\alpha)}}\right)^{[(1+\omega) / 2]}\left(\frac{z}{\left(1+\mathrm{e}^{\mathrm{i} \theta} z\right)^{2(1-\alpha)}}\right)^{[(1-\omega) / 2]} \\
& =\frac{z}{\left[\left(1-\mathrm{e}^{\mathrm{i} \theta} z\right)^{1+\omega}\left(1+\mathrm{e}^{\mathrm{i} \theta} z\right)^{1-\omega}\right]^{1-\alpha}} \quad(\theta \in \mathbb{R} ; 0 \leqq \omega \leqq 1) .
\end{aligned}
$$

Finally, when $\tau=\sigma_{2}$, the equality holds true if and only if $J_{\left(\lambda_{p}\right),\left(\tau_{q}\right), b}^{s, \lambda}(f)(z)$ satisfies the following condition:

$$
\frac{z\left(\lambda_{(\lambda p),\left(\tau_{q}\right), b}^{s, a, \lambda}\right)^{\prime}(f)(z)}{J_{(\lambda, p),\left(\tau_{q}\right), b}^{s, a, \lambda}(f)(z)}=(1-\alpha) p(z)+\alpha
$$

where

$$
\frac{1}{p(z)}=\left(\frac{1+\omega}{2}\right)\left(\frac{1+z}{1-z}\right)+\left(\frac{1-\omega}{2}\right)\left(\frac{1-z}{1+z}\right) \quad(0<\omega<1) .
$$

We conclude this paper by mentioning that, by suitably specializing the parameters involved, our main results (Theorem 1 to Theorem 5) would yield a number of (known or 
new) results for much simpler function classes, which were investigated in several earlier works by employing many special cases of the new generalized Srivastava-Attiya operator.

\section{Competing interests}

The authors declare that they have no competing interests.

\section{Authors' contributions}

All authors completed the paper together. All authors read and approved the final manuscript.

\section{Author details}

'Department of Mathematics and Statistics, University of Victoria, Victoria, British Columbia V8W 3R4, Canada.

2Department of Mathematics and Computer Science, University of Québec at Chicoutimi, Chicoutimi, Québec G7H 2B1,

Canada.

Received: 30 September 2014 Accepted: 22 January 2015 Published online: 03 February 2015

\section{References}

1. Srivastava, HM, Attiya, AA: An integral operator associated with the Hurwitz-Lerch zeta function and differential subordination. Integral Transforms Spec. Funct. 18, 207-216 (2007)

2. Cho, NE, Kim, IH, Srivastava, HM: Sandwich-type theorems for multivalent functions associated with the Srivastava-Attiya operator. Appl. Math. Comput. 217, 918-928 (2010)

3. Răducanu, D, Srivastava, HM: A new class of analytic functions defined by means of a convolution operator involving the Hurwitz-Lerch zeta function. Integral Transforms Spec. Funct. 18, 933-943 (2007)

4. Srivastava, HM, Răducanu, D, Sălăgean, GS: A new class of generalized close-to-starlike functions defined by the Srivastava-Attiya operator. Acta Math. Sin. Engl. Ser. 29, 833-840 (2013)

5. Srivastava, HM, Choi, J: Series Associated with Zeta and Related Functions. Kluwer Academic, Dordrecht (2001)

6. Srivastava, HM: Some formulas for the Bernoulli and Euler polynomials at rational arguments. Math. Proc. Camb. Philos. Soc. 129, 77-84 (2000)

7. Srivastava, HM, Choi, J: Zeta and q-Zeta Functions and Associated Series and Integrals. Elsevier, Amsterdam (2012)

8. Srivastava, HM: A new family of the $\lambda$-generalized Hurwitz-Lerch zeta functions with applications. Appl. Math. Inf. Sci. $8,1485-1500(2014)$

9. Srivastava, HM: Some generalizations and basic (or q-) extensions of the Bernoulli, Euler and Genocchi polynomials. Appl. Math. Inf. Sci. 5, 390-444 (2011)

10. Srivastava, HM: Generating relations and other results associated with some families of the extended Hurwitz-Lerch zeta functions. SpringerPlus 2, Article ID 67 (2013)

11. Srivastava, HM, Gaboury, S: New expansion formulas for a family of the $\lambda$-generalized Hurwitz-Lerch zeta functions. Int. J. Math. Math. Sci. 2014, Article ID 131067 (2014)

12. Srivastava, HM, Jankov, D, Pogány, TK, Saxena, RK: Two-sided inequalities for the extended Hurwitz-Lerch zeta function. Comput. Math. Appl. 62, 516-522 (2011)

13. Srivastava, HM, Saxena, RK, Pogány, TK, Saxena, R: Integral and computational representations of the extended Hurwitz-Lerch zeta function. Integral Transforms Spec. Funct. 22, 487-506 (2011)

14. Srivastava, HM, Manocha, HL: A Treatise on Generating Functions. Halsted, New York (1984)

15. Mathai, AM, Saxena, RK, Haubold, HJ: The H-Function: Theory and Applications. Springer, New York (2010)

16. Srivastava, HM, Gupta, KC, Goyal, SP: The H-Functions of One and Two Variables with Applications. South Asian Publishers, New Delhi (1982)

17. Prajapat, JK, Bulboacă, T: Double subordination preserving properties for a new generalized Srivastava-Attiya operator. Chin. Ann. Math. 33, 569-582 (2012)

18. Noor, KI, Bukhari, SZH: Some subclasses of analytic and spiral-like functions of complex order involving the Srivastava-Attiya integral operator. Integral Transforms Spec. Funct. 21, 907-916 (2010)

19. Choi, JH, Saigo, M, Srivastava, HM: Some inclusion properties of a certain family of integral operators. J. Math. Anal. Appl. 276, 432-445 (2002)

20. Cho, NE, Srivastava, HM: Argument estimation of certain analytic functions defined by a class of multiplier transformation. Math. Comput. Model. 37, 39-49 (2003)

21. Jung, IB, Kim, YC, Srivastava, HM: The Hardy space of analytic functions associated with certain one-parameter families of integral operators. J. Math. Anal. Appl. 176, 138-147 (1993)

22. Bernardi, SD: Convex and starlike univalent functions. Trans. Am. Math. Soc. 135, 429-446 (1969)

23. Carlson, BC, Shaffer, DB: Starlike and prestarlike hypergeometric functions. SIAM J. Math. Anal. 15, 737-745 (1984)

24. Owa, S, Srivastava, HM: Univalent and starlike generalized hypergeometric functions. Can. J. Math. 39, 1057-1077 (1987)

25. Dziok, J, Srivastava, HM: Classes of analytic functions associated with the generalized hypergeometric function. Appl. Math. Comput. 103, 1-13 (1999)

26. Dziok, J, Srivastava, HM: Certain subclasses of analytic functions associated with the generalized hypergeometric function. Integral Transforms Spec. Funct. 14, 7-18 (2003)

27. Hohlov, YE: Operators and operations in the class of univalent functions. Izv. Vysš. Učebn. Zaved., Mat. 10, 83-89 (1978)

28. Ruscheweyh, S: New criteria for univalent functions. Proc. Am. Math. Soc. 49, 109-115 (1975)

29. Duren, PL: Univalent Functions. Grundlehren der Mathematischen Wissenschaften, vol. 259. Springer, Berlin (1983)

30. Ma, WC, Minda, D: A unified treatment of some special classes of functions. In: Proceedings of the Conference on Complex Analysis (Tianjin, 1992). Conf. Proc. Lecture Notes in Anal., vol. 1, pp. 157-169. International Press, Cambridge (1994) 